\title{
Eradication of Helicobacter pylori Induces Immediate Regressive Changes in Early Gastric Adenocarcinomas
}

\author{
Mitsuru Nakagawa ${ }^{a}$ Yasuhiro Sakai $^{a}$ Yuka Kiriyama ${ }^{a}$ b Tomomitsu Taharac \\ Noriyuki Horiguchic Asako Okabe ${ }^{\mathrm{c}}$ Sayumi Tahara ${ }^{\mathrm{a}}$ Tomoyuki Shibata $^{\mathrm{c}}$ \\ Naoki Ohmiyac Makoto Kuroda ${ }^{\mathrm{c}}$ Atsushi Sugiokad Tetsuya Tsukamoto ${ }^{\mathrm{a}}$ \\ a Department of Diagnostic Pathology, Fujita Health University School of Medicine, Toyoake, Japan; \\ ${ }^{b}$ Department of Diagnostic Pathology, Narita Memorial Hospital, Toyohashi, Japan; ' Department of Gastroenterology, \\ Fujita Health University School of Medicine, Toyoake, Japan; ${ }^{d}$ Department of Surgery, Fujita Health University \\ School of Medicine, Toyoake, Japan
}

\author{
Keywords \\ Helicobacter pylori · Eradication · Regression - Proliferative \\ zone
}

\begin{abstract}
Objective: Helicobacter pylori eradication is expected to prevent gastric cancer. However, morphological alterations after eradication often hinder accurate diagnosis. Therefore, we evaluated endoscopic and histological changes in gastric tumors after eradication of $H$. pylori in a time-dependent manner. Methods: We classified 144 cases of endoscopic submucosal dissection (ESD) of early gastric cancer into the following categories: (i) patients positive for $H$. pylori with no eradication history, (ii) patients positive for H. pylori who underwent ESD 2 months after eradication, (iii) patients negative for H. pylori with an eradication history of at least 6 months before ESD, and (iv) patients negative for $H$. pylori with an unknown history. We compared endoscopic and histological factors between the groups. Results: The characteristics of cancers positive for $\mathrm{H}$. pylori were exploding shape, superficial high-grade atypical epithelium, and a surface proliferating zone. H. pylori eradication induced a series of endoscopic and histological changes, including shape
\end{abstract}

(c) 2019 S. Karger AG, Basel

\section{E-Mail karger@karger.com www.karger.com/pat \\ KARGER}

depression, appearance of surface regenerative and lowergrade atypical epithelium, and a downward shift of the proliferative zone within a period as short as 2 months. Conclusion: H. pylori eradication rapidly causes cancer regression and leads to tumor shrinkage, diminished atypism, and shortened proliferative zone, resulting in drastic morphological changes.

(c) 2019 S. Karger AG, Basel

\section{Introduction}

Gastric cancer remains a prevalent cause of diseaserelated deaths worldwide [1]. Helicobacter pylori has been identified as a causative factor for gastric disorders [2], and its infection has been widely accepted as a carcinogenic factor [3]. Epidemiological and experimental studies have strongly indicated that $H$. pylori eradication markedly reduces the risk of gastric disorders [4], and health insurance programs have confirmed the efficacy of this endeavor in preventing metachronous primary gastric cancers [5]. Although there have been negative reports, especially for patients with severe atrophic and metaplastic gastritis [6-8], a recent meta-analysis has 
confirmed the effect of eradication [9]. Thus, it is important to ascertain the morphological changes in gastric cancers detected after successful eradication of $H$. pylori compared to those arising from an underlying active infection.

Several clinical and histopathological studies have indicated that the gastric cancers detected after eradication of $H$. pylori not only display poor demarcation and lack typical endoscopic cancerous characteristics [1014], but they also show a pathologically lower-grade atypia of the neoplastic epithelium $[12,14,15]$, making a precise diagnosis difficult. In the present study, we characterized the changes that occur in gastric cancers immediately and up to several years after $H$. pylori eradication, in order to understand the changes in tumor characteristics over time.

\section{Materials and Methods}

\section{Patients}

We included 144 patients who underwent endoscopic submucosal dissection (ESD) owing to early gastric cancer (pT1a and pT1b) at the Endoscopy Center of Fujita Health University Hospital between 2008 and 2015.

\section{Eradication of H. pylori}

H. pylori infection was detected through immunohistochemical analysis using anti-H. pylori polyclonal antibody (Dako, Glostrup, Denmark) from biopsy specimens, ${ }^{13} \mathrm{C}$-urea breath test with UBIT tablets (Otsuka Pharmaceutical, Tokyo, Japan), and/ or serum IgG titer against $H$. pylori. When at least one of the tests was positive, we administered standardized eradication therapy (30 mg of lansoprazole, $200 \mathrm{mg}$ of clarithromycin, and $750 \mathrm{mg}$ of amoxicillin, twice a day for 1 week) to the patient [10]. Subsequently, the infection status was reevaluated, and the eradication was considered successful when all the tests yielded negative results.

\section{Immunohistochemical Analysis}

We performed immunohistochemistry for the following: MUC5AC (CLH2, Novocastra, Newcastle, UK), MUC6 (CLH5, Novocastra), and CDX2 (CDX2-88, BioGenex, San Ramon, CA, USA) to perform gastric and/or intestinal phenotyping; Ki-67 (MIB-1, Dako) to reveal proliferative activity; p53 (DO-7, Novocastra) to detect abnormal expression due to mutation; and H. pylori (anti-H. pylori polyclonal antibody, Dako). HER2 gene amplification was evaluated using immunohistochemistry using 4B5 (Roche Diagnostics, Basel, Switzerland) or with fluorescent in situ hybridization employing the PathVysion ${ }^{\circledR}$ HER2 DNA Probe Kit (Abbott Laboratories, Abbott Park, IL, USA). All immunohistochemical analyses were performed using the iView DAB Universal Kits run on Ventana Benchmark Ultra (Roche).
Patient Grouping Based on H. pylori Infection Status

Patients were classified based on their infection status and the times of $H$. pylori eradication (Table 1):

(i) Non-eradicated (non-E) group $(n=76)$ : patients who did not undergo eradication therapy previously and were simultaneously diagnosed with early gastric cancer and $H$. pylori infection; these patients were treated with ESD without eradication.

(ii) Non-eradicated at diagnosis but eradicated before ESD (preoperatively eradicated, POE) group $(n=10)$ : these patients shared a background similar to that of the non-E patients, but $H$. pylori was eradicated up to 2 months before ESD (Table 2).

(iii) Already eradicated at diagnosis (prediagnostically eradicated, PDE) group $(n=43)$ : for these patients, the infection was eradicated when the cancer was diagnosed endoscopically. This group was further divided into two subgroups based on the timing of cancer detection: between 6 and 36 months after eradication $(n=11$, PDE-early) and beyond 36 months $(n=25$, PDElate) (Table 2). Seven cases, for which the eradication treatment date was not precisely recorded, were excluded from the trend analysis.

(iv) Spontaneously eradicated (SE) group $(n=15)$ : although $H$. pylori tests were negative for these patients with no history of $H$. pylori eradication, endoscopic findings of infection such as corpus atrophic or metaplastic gastritis, and gastric cancer, were identified. Spontaneous eradication due to past antibiotic therapy may have been the underlying cause.

Endoscopic and histological findings in the non-E and PDE groups were reflected by $H$. pylori-positive and -negative gastric backgrounds, respectively. In the POE group, these findings were compatible with ongoing histopathological changes following $H$. pylori eradication, because the cancer was dissected within 2 months after eradication.

Endoscopic and Histopathological Evaluation of Gastric Tumors

The location of the tumor in the stomach was anatomically divided into 3 sections: upper, middle, and lower.

According to the Paris endoscopic classification of superficial neoplastic lesions and the Japanese classification of gastric cancers, the morphology of the tumor was classified as "protruded/elevated" or "flat/depressed." The former consisted of 0-I (protruded type), 0-IIa (superficially elevated type), and 0-IIa + IIc (superficially elevated, but partially depressed type). The latter was compatible with 0 -IIb (flat type), 0-IIc (superficially depressed type), and 0-III (excavated type) (Table 1) [16, 17].

The microscopic shape of the tumor was sorted into 4 types: "polypoid," "superiorly exploding," "intermediate," and "laterally infiltrating" (Fig. 1). Superiorly exploding tumor is a bowl-shaped tumor that expands more proliferously in the upper layer than in the lower layer. Conversely, laterally infiltrating tumor is a roundedged tumor that spreads laterally in the middle layer. The intermediate type has an appearance between that of the superiorly exploding and laterally infiltrating types.

Histopathological grading of the tumor was performed based on the World Health Organization's criteria for the classification of digestive system tumors [18].

Based on the microscopic findings, the surface epithelium over the tumor was categorized as follows: (i) regenerative columnar epithelium (RCE), (ii) epithelium with low-grade atypia (ELA), (iii) a combination of RCE and ELA, and (iv) epithelium with highgrade atypia (EHA) [12]. 
Table 1. The clinical and endoscopic characteristics of the patients

\begin{tabular}{|c|c|c|c|c|c|c|}
\hline & Total & $\begin{array}{l}\text { Non-E } \\
\text { (non-eradicated) }\end{array}$ & $\begin{array}{l}\text { POE } \\
\text { (preoperatively } \\
\text { eradicated) }\end{array}$ & $\begin{array}{l}\text { PDE } \\
\text { (prediagnostically } \\
\text { eradicated) }\end{array}$ & $\begin{array}{l}\text { SE } \\
\text { (spontaneously } \\
\text { eradicated) }\end{array}$ & $\begin{array}{l}p \\
\text { values }^{\mathrm{a}}\end{array}$ \\
\hline Sex & & & & & & 0.293 \\
\hline Male & 111 & 63 & 8 & 30 & 10 & \\
\hline Age (range, median), years & $53-92(72.0)$ & $53-89(70.5)$ & $61-87(72.5)$ & $53-87(74.0)$ & $68-92(75.0)$ & 0.219 \\
\hline Location & & & & & & 0.691 \\
\hline Upper (fundus) & 17 & 7 & 2 & 6 & 2 & \\
\hline Middle (corpus) & 55 & 32 & 4 & 16 & 3 & \\
\hline Lower (antrum/pylorus) & 72 & 37 & 4 & 21 & 10 & \\
\hline $\begin{array}{l}\text { Endoscopic maximum } \\
\quad \text { diameter (mean } \pm S D), \mathrm{mm}\end{array}$ & $12.47 \pm 6.48$ & $12.79 \pm 5.56$ & $13.50 \pm 4.20$ & $11.21 \pm 7.82^{\mathrm{b}}$ & $14.00 \pm 7.57$ & 0.029 \\
\hline
\end{tabular}

Table 2. The interval between endoscopic submucosal dissection and Helicobacter pylori eradication

\begin{tabular}{|c|c|c|c|c|}
\hline & Non-E $(n=76)$ & $\operatorname{POE}(n=10)$ & PDE-early $(n=11)$ & PDE-late $(n=25)$ \\
\hline Interval between HP eradication and ESD & 0 & $0-2$ months & $6-36$ months & $36-180$ months \\
\hline Mean \pm SD & NA & $33.6 \pm 12.3$ days & $18.4 \pm 8.7$ months & $90.6 \pm 48.1$ months \\
\hline Range & NA & $19-56$ days & 6-34 months & $36-180$ months \\
\hline
\end{tabular}

HP, Helicobacter pylori; ESD, endoscopic submucosal dissection; non-E, non-eradication; POE, preoperatively eradicated; PDE, prediagnostically eradicated; SD, standard deviation; NA, not applicable.

The phenotype was identified based on the expression of MUC5AC, MUC6, and CDX2. MUC5AC- or MUC6-positive tumors were classified as "gastric," while CDX2-positive tumors were classified as "intestinal." Tumors with both markers were categorized as "gastrointestinal," while those harboring neither of the markers were placed under the "null" phenotype [19]. Distinct staining of $>5 \%$ of the tumor cells was considered positive.

The proliferative zone of the tumor was alternatively evaluated according to the Ki-67-positive tumor cell distribution and categorized into the "upper layer" when the positive cells were distributed in and beneath the surface epithelium, or into the "middle layer" when the positive cells were located in the middle of the neoplastic mucosa.

Eradicated Gastric Cancer Regression p53 mutation was speculated in the event of a positive cell ratio for its protein, determined using immunohistochemistry. We defined the "wild type pattern" as $1-33 \%$ positivity and the "aberrant pattern" as $<1 \%$ or $>33 \%$ positivity.

HER2 scoring was performed based on guidance provided in a previous report [20].

\section{Statistical Analysis}

Differences among the groups were analyzed using the Kruskal-Wallis and Dunn's multiple comparison tests. To discern the association between the categorical variables in the contingency tables, Pearson's $\chi^{2}$ test and Harberman's residual analysis were applied when all expected values were $>1.0$ and at least $20 \%$ of the 

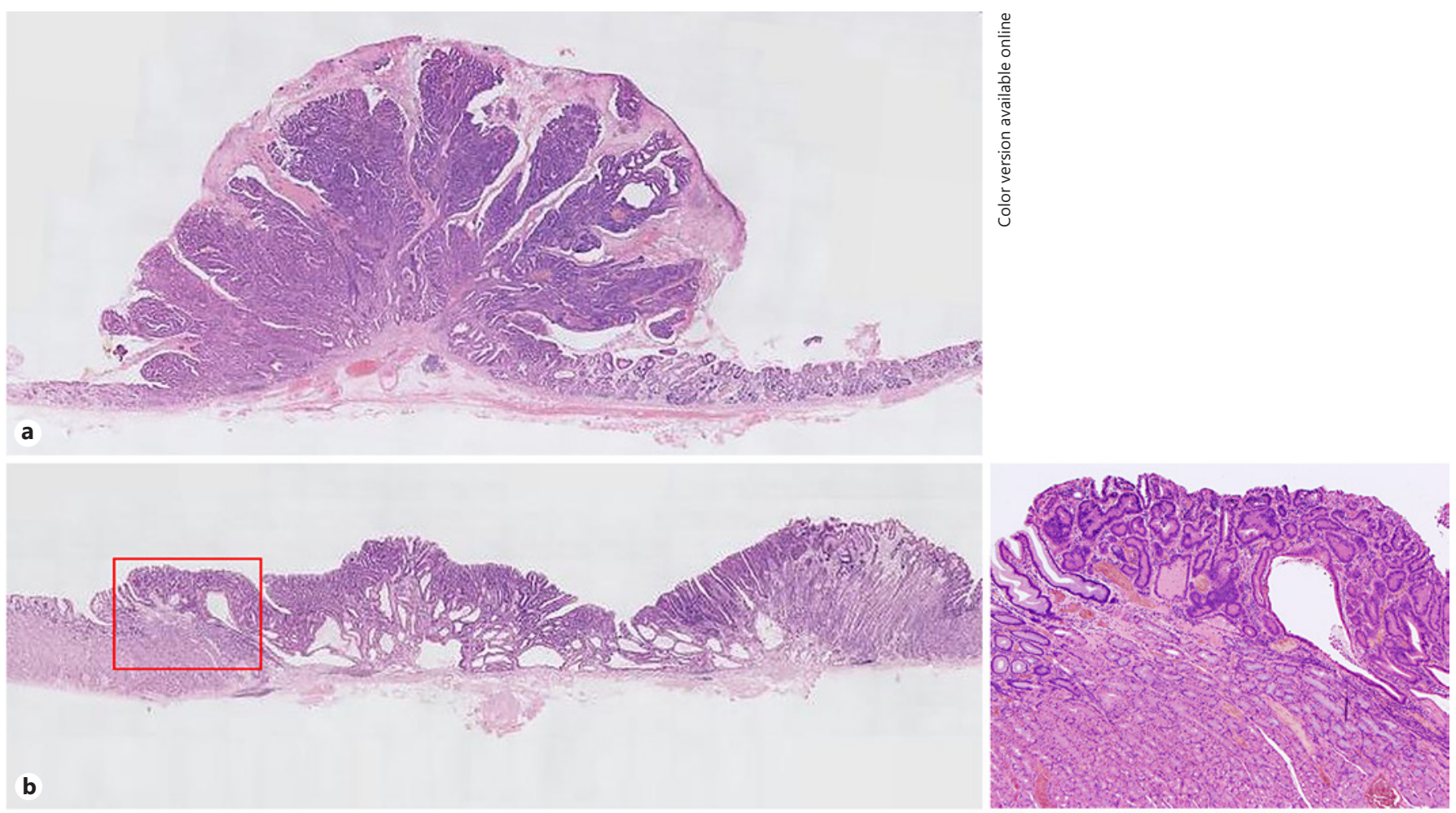

b
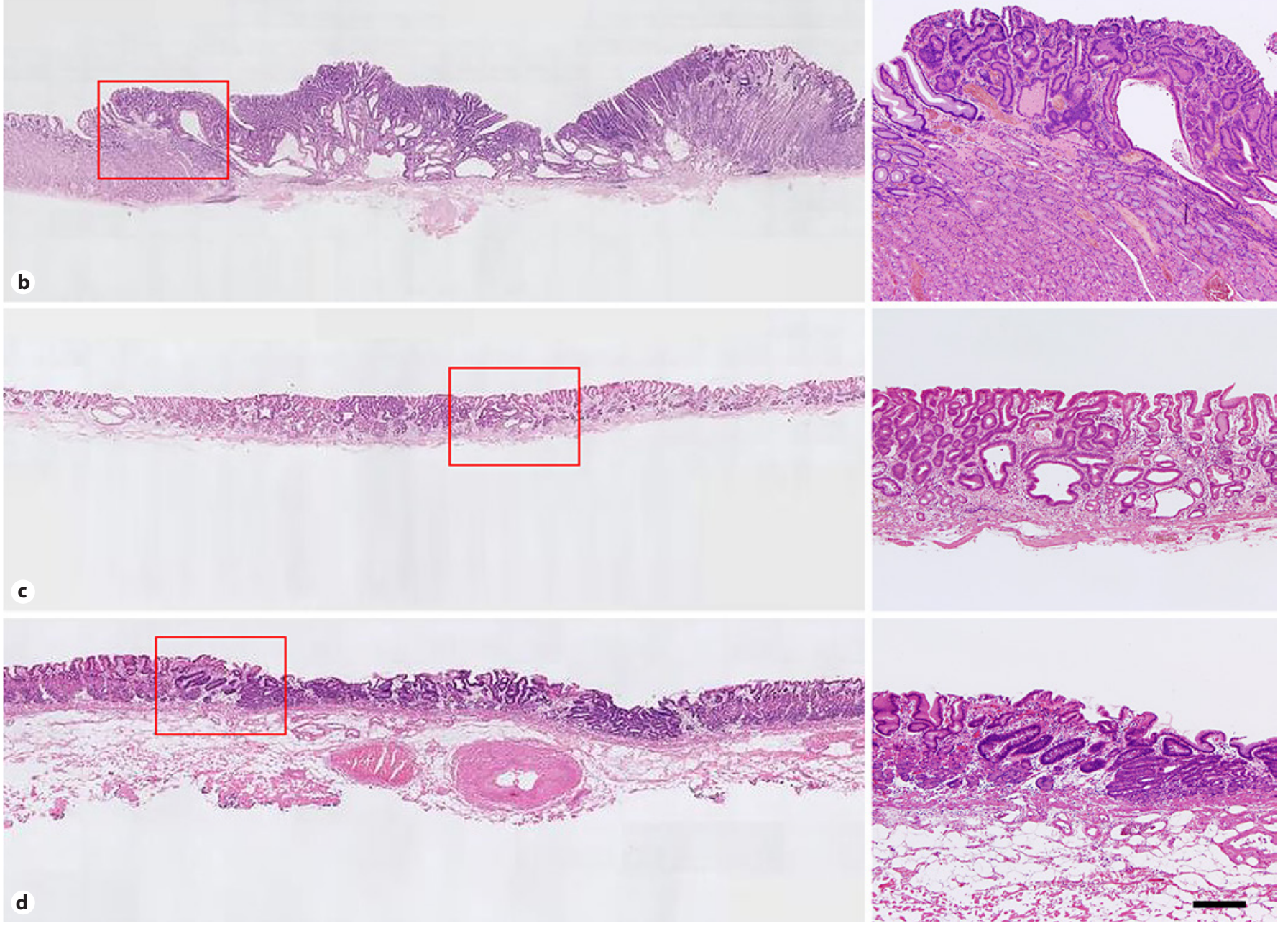

Fig. 1. The 4 microscopic shapes of gastric adenocarcinoma. a Polypoid type is a tumor that forms a polypoid shape. b Superiorly exploding type is a bowl-shaped tumor that expands more proliferously in the upper layer than in the lower layer of the tumor. c Intermediate type is a tumor with an uneven border between the tumor and background mucosa, showing an intermediate position between superior exploding and laterally infiltrating types. $\mathbf{d}$ Laterally infiltrating type is a round-edged tumor that laterally spreads through the middle layer of the tumor. Hematoxylin and eosin staining. Bar, $600 \mu \mathrm{m}$ for left panels and $200 \mu \mathrm{m}$ for right panels. 


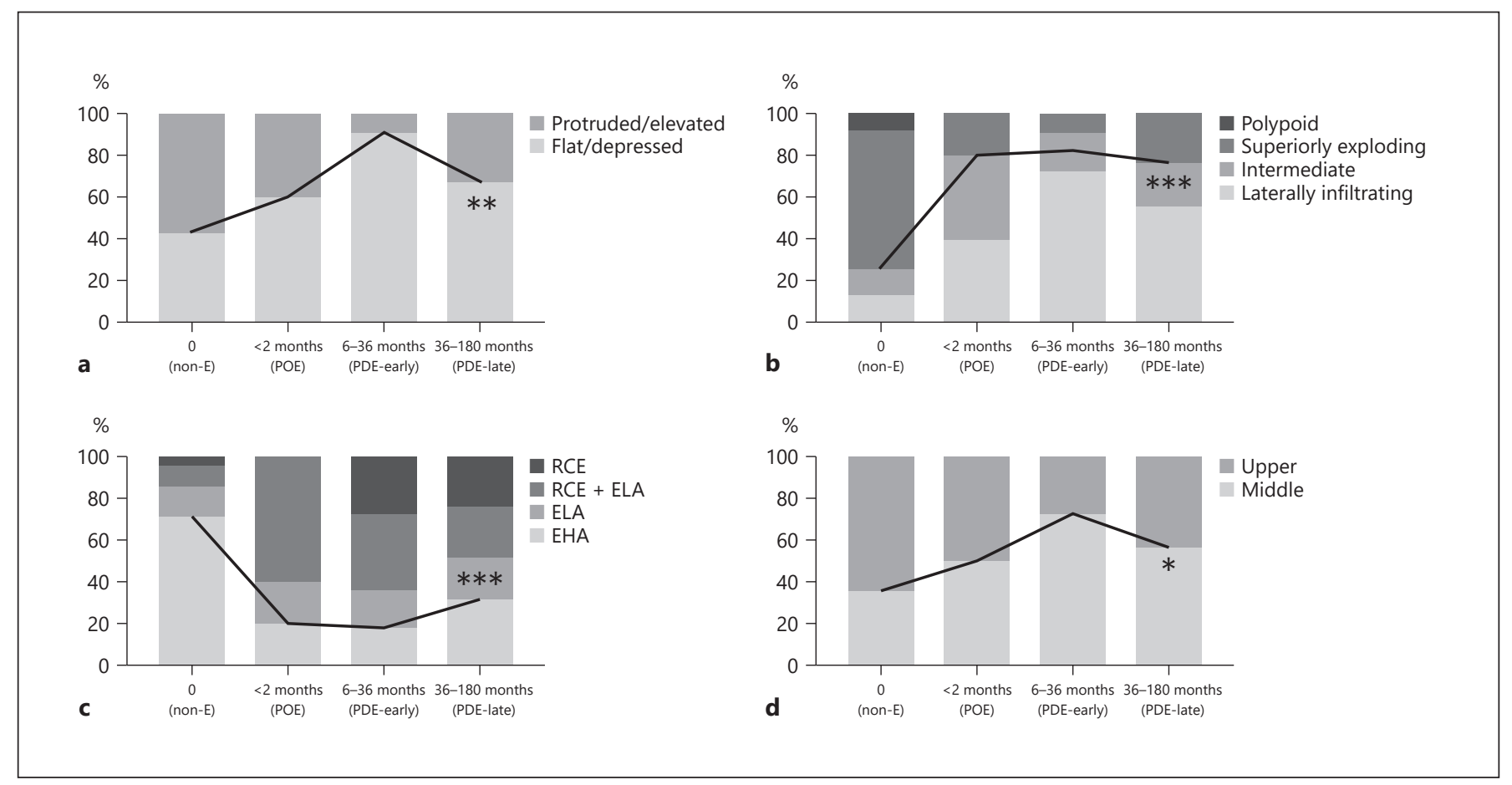

Fig. 2. Trend analyses for endoscopic morphology (a), microscopic shape (b), surface epithelium over the tumor (c), and proliferating zone (d) over time after eradication for the non-eradicated (non-E) group, the preoperatively eradicated (POE) group, and the prediagnostically eradicated (PDE) group. The protruded/elevated endoscopic morphology, superiorly exploding microscopic shape, epithelium with high-grade atypia (EHA) in the surface above the tumor, and a proliferating zone located in the upper layer were all observed more frequently in non-E group cancer. Serial endoscopic and histopathological changes to the flat/depressed and laterally infiltrating type, regenerative columnar epithelium (RCE) and epithelium with low-grade atypia (ELA) in the surface, and a downward-proliferating zone were noted gradually over time after eradication with statistical significance. ${ }^{*} p<0.05,{ }^{* *} p<0.01$, and ${ }^{* * *} p<0.001$ for the Cochran-Armitage trend test. expected values were $>5$. Otherwise, Yates' correction for continuity was adjusted to Pearson's $\chi^{2}$ test (Yates' $\chi^{2}$ test). Trend analysis was performed using the Cochran-Armitage test. Kruskal-Wallis and Dunn's multiple comparison tests and the Cochran-Armitage tests were evaluated using GraphPad Prism 7 software (GraphPad Software, La Jolla, CA, USA). $p<0.05$ was considered statistically significant.

\section{Results}

\section{Differences in Endoscopic and Histopathological}

Appearances Based on the H. pylori Infection Status

The clinical and endoscopic characteristics of gastric cancers for the 4 patient groups are summarized in Table 1 . No statistically significant differences were observed in sex, age, and location of the tumor. However, Harberman's residual analysis following Pearson's $\chi^{2}$ test revealed that the protruded/elevated and flat/depressed types were more common in the non-E (adjusted standardized residual, ASR $=2.338, p=0.018)$ and $\mathrm{PDE}$ groups (ASR $=3.029, p=0.003$ ), respectively. The Cochran-Armitage trend test indicated that the endoscopic morphology shifted from the protruded/elevated type to the flat/depressed type over time after eradication $(p=$ 0.009) (Fig. 2a). Dunn's multiple comparison test following the Kruskal-Wallis test revealed that the maximum diameter of the tumor was significantly smaller in the PDE group than in the non-E group $(p=0.048)$. These findings suggest that posteradication gastric cancer becomes smaller, flatter, and more depressed than $H$. pyloriassociated cancer.

The histopathological findings are summarized in Table 3. Pearson's and Yates' $\chi^{2}$ test ascertained that significant differences in the microscopic shape, surface epithelium over the tumor, and proliferating zone existed among the groups. Nonetheless, no significant differenc- 
Fig. 3. Representative cases of cancer in the non-eradicated (non-E) and prediagnostically eradicated (PDE) groups. The tumor grows toward the superficial lumen in the non-E group, whereas the tumor spreads laterally in the middle layer in the PDE group, and the surface is covered with regenerative normal surface foveolar epithelium and pyloric glands. The Ki-67-labeled proliferative zone is located in the upper and middle layers in non-E and PDE group cancers, respectively. We evaluated one case each of non-E and PDE group cancers as the p53 aberrant pattern and the wildtype pattern, respectively. Both tumors show CDX2+, MUC5AC-, and MUC6intestinal phenotypes. $\mathrm{HE}$, hematoxylin and eosin staining. Bar, $200 \mu \mathrm{m}$.
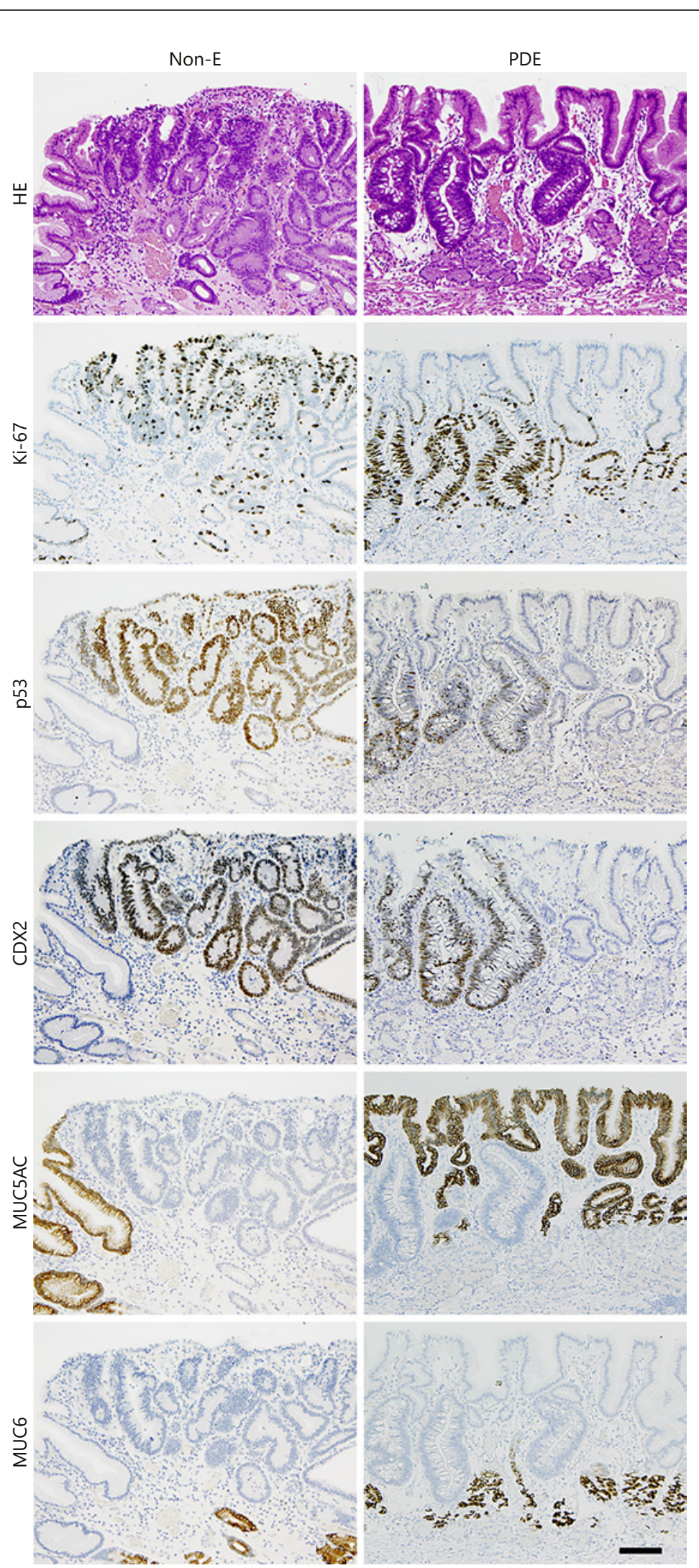
Table 3. Histological findings of gastric cancers classified based on the Helicobacter pylori infection status

\begin{tabular}{|c|c|c|c|c|c|c|}
\hline \multirow{3}{*}{$\begin{array}{l}\text { At clinical diagnosis: } \\
\text { At pathological sampling: }\end{array}$} & \multicolumn{2}{|l|}{$\mathrm{HP}+$} & \multicolumn{2}{|l|}{$\mathrm{HP}-$} & \multirow{3}{*}{$\begin{array}{l}\text { Total } \\
(n=144)\end{array}$} & \multirow[t]{3}{*}{$p$ value ${ }^{a}$} \\
\hline & \multirow{2}{*}{$\begin{array}{l}\mathrm{HP}+ \\
\text { non-E } \\
(n=76)\end{array}$} & \multicolumn{3}{|l|}{$\mathrm{HP}-$} & & \\
\hline & & $\begin{array}{l}\mathrm{POE} \\
(n=10)\end{array}$ & $\begin{array}{l}\mathrm{PDE} \\
(n=43)\end{array}$ & $\begin{array}{l}\mathrm{SE} \\
(n=15)\end{array}$ & & \\
\hline \multicolumn{7}{|l|}{ Microscopic shape } \\
\hline Polypoid & 6 & 0 & 0 & 1 & 7 & $<0.001$ \\
\hline Superiorly exploding & $50^{* * *}$ & 2 & $8^{* * *}$ & 9 & 69 & \\
\hline Intermediate & 10 & $4^{*}$ & 6 & 3 & 23 & \\
\hline Laterally infiltrating & $10^{* * *}$ & 4 & $29 * * *$ & 2 & 45 & \\
\hline Histological type & & & & & & 0.200 \\
\hline Tubular & 76 & 10 & 42 & 13 & 141 & \\
\hline Papillary & 0 & 0 & 0 & 2 & 2 & \\
\hline Poorly cohesive & 0 & 0 & 1 & 0 & 1 & \\
\hline Depth of invasion & & & & & & 0.999 \\
\hline pT1a (mucosa) & 69 & 9 & 38 & 14 & 130 & \\
\hline pT1b (submucosa) & 7 & 1 & 5 & 1 & 14 & \\
\hline Surface epithelium over the tumor & & & & & & $<0.001$ \\
\hline $\mathrm{RCE}$ & $3^{* *}$ & 0 & $10^{* *}$ & 3 & 16 & \\
\hline ELA & 11 & 2 & $14^{* *}$ & 1 & 28 & \\
\hline $\mathrm{RCE}+\mathrm{ELA}$ & 8 & $6 * * *$ & 8 & 0 & 22 & \\
\hline EHA & $54^{* * *}$ & $2^{*}$ & $11^{* * *}$ & 11 & 78 & \\
\hline Phenotype & & & & & & 0.546 \\
\hline Gastric & 15 & 2 & 9 & 8 & 34 & \\
\hline Gastrointestinal & 35 & 5 & 22 & 2 & 64 & \\
\hline Intestinal & 23 & 3 & 11 & 5 & 42 & \\
\hline Null & 3 & 0 & 1 & 0 & 4 & \\
\hline Proliferating zone (Ki-67) & & & & & & 0.021 \\
\hline Upper layer & $49^{* *}$ & 5 & $15^{* *}$ & 8 & 77 & \\
\hline Middle layer & $27^{* *}$ & 5 & $28^{* *}$ & 7 & 67 & \\
\hline p53 immunoreactivity & & & & & & 0.746 \\
\hline Normal & 46 & 5 & 22 & 8 & 81 & \\
\hline Aberrant & 30 & 5 & 21 & 7 & 63 & \\
\hline HER2 gene amplification & & & & & & 0.770 \\
\hline Yes & 6 & 1 & 3 & 3 & 13 & \\
\hline No & 70 & 9 & 40 & 12 & 131 & \\
\hline
\end{tabular}

a Assessed by $\chi^{2}$ test. ${ }^{*} p<0.05,{ }^{* *} p<0.01,{ }^{* * *} p<0.001$ (Harberman's residual analysis). HP, Helicobacter pylori; non-E, non-eradicated; POE, preoperatively eradicated; PDE, prediagnostically eradicated; SE, spontaneously eradicated; RCE, regenerative columnar epithelium; ELA, epithelium with low-grade atypia; EHA, epithelium with high-grade atypia.

es were noted in the histopathological type, depth of invasion, phenotype, p53 immunoreactivity, and HER2 gene amplification (Fig. 3).

\section{Serial Changes in the Microscopic Shape from}

Superiorly Exploding to Laterally Infiltrating Type

With respect to the microscopic shape, 65.8\% (50/76) of the non-E group cancers were of the superiorly exploding type, whereas $67.4 \%$ (29/43) of the PDE group cancers belonged to the laterally infiltrating type (Table 3). Based on statistical analysis, the most common tumor types were the superiorly exploding type in the non-E group (ASR = $4.539, p<0.001$ ), the intermediate type in the POE group $(\mathrm{ASR}=2.150, p=0.032)$, and the laterally infiltrating type in the PDE group (ASR $=6.114, p<0.001)$. Laterally infiltrating tumors in the non-E group (ASR $=-4.952, p<$ 
$0.001)$ and superiorly exploding tumors in the PDE group $(\mathrm{ASR}=-4.594, p<0.001)$ manifested themselves to a lesser extent. The tumors were broadly divided into two categories: polypoid/superiorly exploding and intermediate/ laterally infiltrating. This classification revealed a trend, wherein the latter type was most frequently manifested in the PDE (particularly the PDE-early) group, followed in order by the POE and non-E groups, with statistical significance ( $p<0.001$, Fig. $2 b)$. These findings suggest that the microscopic shape of gastric cancer may change from the superiorly exploding to laterally infiltrating type over time after eradication treatment.

Gradual Decrease of Atypia in the Surface Epithelium over the Tumor

EHA was observed in the surface over the tumor as well as in the cancer itself in 71.0\% (54/76) of the non-E group, while the surface epithelium displayed milder atypia, such as RCE, ELA, or both, in $74.4 \%(32 / 43)$ of the PDE group (Table 3). With statistical significance, EHA $(\mathrm{ASR}=4.299 . p<0.001), \mathrm{RCE}+\mathrm{ELA}(\mathrm{ASR}=4.075, p<$ $0.001)$, and either RCE (ASR $=3.026, p=0.002$ ) or ELA (ASR $=2.594, p=0.009)$ were more frequently observed above the tumor in the non-E, POE, and PDE groups, respectively. Trend analysis revealed that the atypia gradually became milder, while the regenerative change became more prominent in the surface epithelium over time after eradication ( $p<0.001$, Fig. $2 c)$. Thus, the regenerative and low-grade epithelium may become proliferously covered with the tumor after the eradication of $H$. pylori infection.

\section{Downward Shifting of the Ki-67-Labeled Proliferating Zone}

The Ki-67-labeled proliferative zone was frequently observed in the upper layer of the neoplastic mucosa in the non-E group (ASR $=2.798, p=0.005)$, whereas it was often distributed in the middle layer in the PDE group (ASR $=2.918, p=0.004$ ), with statistical significance. Trend analysis comparing the patient groups revealed that the proliferative zone moved downward in the neoplastic mucosa as the time from eradication increased $(p=0.021$, Fig. 2d).

\section{Discussion}

This study demonstrates the differences between $H$. pylori-eradicated and non-eradicated gastric cancers in not only the endoscopic morphology and size, but also in the histopathological characteristics. We also show the gradual endoscopic and histopathological changes over time after $H$. pylori eradication treatment.

Endoscopic observations revealed that the maximum diameter in the H. pylori-negative (PDE) group was smaller than that in the positive (non-E) group. Moreover, the morphology of gastric cancer shifted gradually from protruded/elevated to flat/depressed upon $H$. pylori eradication. These results are compatible with those of previous studies that reported a decrease in the height of superficially elevated tumors and the fading of the demarcation line 1 month after successful eradication [15]. Furthermore, the tumor size was significantly smaller in patients who had received eradication therapy before cancer dissection [21-23].

We also found that the superiorly exploding type in the microscopic shape, EHA in the surface above the tumor, and the proliferating zone located in the upper layer were more common in $H$. pylori-positive gastric cancer, while sequential changes to the laterally infiltrating type, RCE and ELA in the surface, and a downwardproliferating zone were noted after eradication. Gastric cancer positive for $H$. pylori was of the superiorly exploding type because the proliferating zone was distributed in the upper layer of the neoplastic mucosa and the tumor was more proliferous in the upper layer facing the lumen than in the lower layer. However, H. pylori-negative gastric cancer manifests itself as the laterally infiltrating type because the proliferating zone is in the middle layer of the neoplastic mucosa, while the regenerative epithelium instead grows in the upper and lower layers. Regenerative change and atypism in the surface epithelium above the tumor may depend on proliferative activity in the upper layer of the tumor $[12,15]$. Thus, the shifting of the proliferative zone from the upper to the middle part of the neoplastic mucosa as a result of $H$. pylori eradication may influence the endoscopic and histopathological characteristics [24].

Herein, the gastric tumors were histopathologically differentiated tubular adenocarcinomas, except for 2 cases of differentiated papillary carcinoma in the SE group and 1 case of poorly cohesive carcinoma in the PDE group. No significant differences were noted in the histopathological types of the eradicated and non-eradicated cancers, and this result was consistent with previous reports $[11,14,22,25,26]$. Additionally, independently of the eradication status, a gastrointestinal phenotype was observed more often than the gastric, intestinal, and null phenotypes in our analysis. However, Yamamoto et al. [26] reported that the gastric phenotype was more com- 
mon than the intestinal phenotype, particularly after eradication. Further studies are needed to address this discrepancy.

Intriguingly, in this study, trend analysis revealed that shrinking, flattening, and depression of the gastric cancer began immediately (within 2 months) after $H$. pylori eradication and reached a plateau after 36 months. The drastic endoscopic and histopathological changes observed after eradication are associated with an endoscopically indistinct alteration called "gastritis-like appearance" [14]. According to the present study, the gastritislike appearance is witnessed even immediately after eradication. We therefore call the attention of endoscopists to the screening of $H$. pylori-eradicated gastric cancer because the early discovery of gastric cancer after eradication may contribute to a better prognosis for the patients [27].

Meanwhile, gastric cancers over 36 months after eradication tended to be slightly reversed in terms of their morphology and proliferative activity. Recent studies have revealed that DNA methylation was decreased in certain genes such as $L O X$, whereas it was unchanged in $A P C$. Methylation status in the MOS gene could be associated with continuous atrophy and intestinal metaplasia after eradication $[28,29]$. It has also been reported that DNA methylation and microsatellite abnormalities are regressed to normal levels in the long term after eradication in some cases $[28,30]$. Regenerative mucosal proliferation could be due to mucosal status whether it had passed the "point of no return" as characterized as intestinal metaplasia or dysplasia [31]. In consequence, the subsequent altered gene expression may lead to development of post-eradication gastric cancers, which could be more proliferative than those from unrecovered mucosa.

Our statistical analyses suggest that the pathological diagnosis of $H$. pylori-eradicated gastric cancer is sometimes challenging due to cancer regression, such as diminished atypism and shortened proliferative zones in the tumor area, particularly in biopsy specimens, although several pathologists have empirically noticed the difficulty. H. pylori infection status is extremely important in pathological diagnosis to prevent overlooking gastric cancer. Hence, pathologists and endoscopists should be careful during the evaluation of $H$. pylori-eradicated gastric cancer.

\section{Acknowledgments}

This study was supported in part by a Grant-in-Aid from the Ministry of Education, Culture, Sports, Science and Technology, Japan (No. 15K08960). We thank Mr. Yutaka Hirasawa, Mr. Yoshiaki Kawashima, Ms. Satomi Ito, and Ms. Maki Fujiwara for their expert technical assistances.

\section{Statement of Ethics}

The study was conducted with approval from the Institutional Review Board of Fujita Health University.

\section{Disclosure Statement}

The authors have no conflicts of interest to declare.

\section{References}

1 Torre LA, Siegel RL, Ward EM, Jemal A. Global cancer incidence and mortality rates and trends-an update. Cancer Epidemiol Biomarkers Prev. 2016 Jan;25(1):16-27.

2 Warren JR, Marshall B. Unidentified curved bacilli on gastric epithelium in active chronic gastritis. Lancet. 1983 Jun;1(8336):1273-5.

3 Uemura N, Okamoto S, Yamamoto S, Matsumura N, Yamaguchi S, Yamakido M, et al. Helicobacter pylori infection and the development of gastric cancer. N Engl J Med. 2001 Sep;345(11):784-9.

4 Fukase K, Kato M, Kikuchi S, Inoue K, Uemura N, Okamoto S, et al.; Japan Gast Study Group. Effect of eradication of Helicobacter pylori on incidence of metachronous gastric carcinoma after endoscopic resection of early gastric cancer: an open-label, randomised controlled trial. Lancet. 2008 Aug;372(9636):392-7.
5 Asaka M, Mabe K. Strategies for eliminating death from gastric cancer in Japan. Proc Jpn Acad, Ser B, Phys Biol Sci. 2014;90(7):251-8.

6 Chen HN, Wang Z, Li X, Zhou ZG. Helicobacter pylori eradication cannot reduce the risk of gastric cancer in patients with intestinal metaplasia and dysplasia: evidence from a metaanalysis. Gastric Cancer. 2016 Jan;19(1):16675.

7 Wong BC, Lam SK, Wong WM, Chen JS, Zheng TT, Feng RE, et al.; China Gastric Cancer Study Group. Helicobacter pylori eradication to prevent gastric cancer in a high-risk region of China: a randomized controlled trial. JAMA. 2004 Jan;291(2): 187-94.
8 Yanaoka K, Oka M, Ohata $\mathrm{H}$, Yoshimura N Deguchi H, Mukoubayashi C, et al. Eradication of Helicobacter pylori prevents cancer development in subjects with mild gastric atrophy identified by serum pepsinogen levels Int J Cancer. 2009 Dec;125(11):2697-703.

9 Sugano K. Effect of Helicobacter pylori eradication on the incidence of gastric cancer: a systematic review and meta-analysis. Gastric Cancer. 2018 Sep 11; Epub ahead of print..

10 Horiguchi N, Tahara T, Kawamura T, Okubo M, Ishizuka T, Nakagawa Y, et al. Distinct clinic-pathological features of early differentiated-type gastric cancers after Helicobacter pylori eradication. Gastroenterol Res Pract. 2016;2016:8230815. 
11 Kamada T, Hata J, Sugiu K, Kusunoki H, Ito M, Tanaka S, et al. Clinical features of gastric cancer discovered after successful eradication of Helicobacter pylori: results from a 9-year prospective follow-up study in Japan. Aliment Pharmacol Ther. 2005 May;21(9):1121-6.

12 Kitamura Y, Ito M, Matsuo T, Boda T, Oka S, Yoshihara M, et al. Characteristic epithelium with low-grade atypia appears on the surface of gastric cancer after successful Helicobacter pylori eradication therapy. Helicobacter. 2014 Aug;19(4):289-95.

13 Okubo M, Tahara T, Shibata T, Nakamura M, Yoshioka D, Maeda Y, et al. Changes in gastric mucosal patterns seen by magnifying NBI during H. pylori eradication. J Gastroenterol. 2011 Feb;46(2):175-82.

14 Saka A, Yagi K, Nimura S. Endoscopic and histological features of gastric cancers after successful Helicobacter pylori eradication therapy. Gastric Cancer. 2016 Apr;19(2):524-30.

15 Ito M, Tanaka S, Takata S, Oka S, Imagawa S, Ueda $\mathrm{H}$, et al. Morphological changes in human gastric tumours after eradication therapy of Helicobacter pylori in a short-term followup. Aliment Pharmacol Ther. 2005 Mar; 21(5):559-66.

16 Participants in the Paris Workshop. The Paris endoscopic classification of superficial neoplastic lesions: esophagus, stomach, and colon: November 30 to December 1, 2002. Gastrointest Endosc. 2003 Dec;58(6Suppl):S3-43.

17 Japanese Gastric Cancer Association. Japanese classification of gastric carcinoma: 3rd English edition. Gastric Cancer. 2011 Jun; 14(2):101-12.

18 Bosman FT, Carneiro F, Hruban RH, Theise ND. WHO Classification of Tumours of the Digestive System. 4th ed. Lyon: IARC Press; 2010.
19 Tsukamoto T, Toyoda T, Mizoshita T, Tatematsu M. Helicobacter pylori infection and gastric carcinogenesis in rodent models. Semin Immunopathol. 2013 Mar;35(2):17790.

20 Bartley AN, Washington MK, Colasacco C, Ventura CB, Ismaila N, Benson AB 3rd, et al. HER2 testing and clinical decision making in gastroesophageal adenocarcinoma: Guideline from the College of American Pathologists, American Society for Clinical Pathology, and the American Society of Clinical Oncology. J Clin Oncol. 2017 Feb;35(4):446-64.

21 Matsuo T, Ito M, Tatsugami M, Boda T, Takata $S$, Tanaka $S$, et al. Gastric cancer development after Helicobacter pylori eradication therapy: a new form of gastric neoplasia. Digestion. 2012;85(1):61-7.

22 Nishizawa T, Suzuki H, Arano T, Yoshida S, Yamashita H, Hata K, et al. Characteristics of gastric cancer detected within 1 year after successful eradication of Helicobacter pylori. J Clin Biochem Nutr. 2016 Nov;59(3):226-30.

23 Gotoda T, Saito D, Kondo H, Ono H, Oda I, Fujishiro M, et al. Endoscopic and histological reversibility of gastric adenoma after eradication of Helicobacter pylori. J Gastroenterol. 1999;34 Suppl 11:91-6.

24 Kobayashi M, Hashimoto S, Nishikura K, Mizuno K, Takeuchi M, Sato Y, et al. Magnifying narrow-band imaging of surface maturation in early differentiated-type gastric cancers after Helicobacter pylori eradication. J Gastroenterol. 2013 Dec;48(12):1332-42.

25 Kim HJ, Kim N, Yoon H, Choi YJ, Lee JY, Kwon YH, et al. Comparison between resectable Helicobacter pylori-negative and -positive gastric cancers. Gut Liver. 2016 Mar; $10(2): 212-9$.
26 Yamamoto K, Kato M, Takahashi M, Haneda M, Shinada K, Nishida U, et al. Clinicopathological analysis of early-stage gastric cancers detected after successful eradication of Helicobacter pylori. Helicobacter. 2011 Jun;16(3): 210-6.

27 Sakitani K, Nishizawa T, Arita M, Yoshida S, Kataoka Y, Ohki D, et al. Early detection of gastric cancer after Helicobacter pylori eradication due to endoscopic surveillance. Helicobacter. 2018 Aug;23(4):e12503.

28 Shin CM, Kim N, Lee HS, Park JH, Ahn S, Kang GH, et al. Changes in aberrant DNA methylation after Helicobacter pylori eradication: a long-term follow-up study. Int J Cancer. 2013 Nov; 133(9):2034-42.

29 Nakajima T, Enomoto S, Yamashita S, Ando T, Nakanishi Y, Nakazawa K, et al. Persistence of a component of DNA methylation in gastric mucosae after Helicobacter pylori eradication. J Gastroenterol. 2010;45(1):3744.

30 Chan AO, Peng JZ, Lam SK, Lai KC, Yuen MF, Cheung HK, et al. Eradication of Helicobacter pylori infection reverses E-cadherin promoter hypermethylation. Gut. 2006 Apr; 55(4):463-8.

31 Kiriyama Y, Tahara T, Shibata T, Okubo M, Nakagawa M, Okabe A, et al. Gastric-and-intestinal mixed intestinal metaplasia is irreversible point with eradication of Helicobacter pylori. Open J Pathol. 2016;6(2):93104 